\title{
Interlayer charge transfer in tin disulphide: Orbital anisotropy and temporal aspects
}

\author{
Fredrik O. L. Johansson $\odot,{ }^{1, *}$ Xin Chen $\odot,{ }^{2}$ Olle Eriksson, ${ }^{2}$ Biplab Sanyal, ${ }^{2}$ and Andreas Lindblad $\odot{ }^{1}$ \\ ${ }^{1}$ Division of Molecular and Condensed Matter Physics, Department of Physics and Astronomy, Uppsala University, \\ Box 516, SE-751 20 Uppsala, Sweden \\ ${ }^{2}$ Division of Materials Theory, Department of Physics and Astronomy, Uppsala University, Box 516, SE-751 20 Uppsala, Sweden
}

(Received 8 May 2020; revised 3 July 2020; accepted 7 July 2020; published 31 July 2020)

\begin{abstract}
An investigation of the unoccupied electronic structure of the transition-metal dichalcogenide tin disulphide has been conducted using core-hole clock spectroscopy. Polarization-dependent X-ray absorption in the tender $\mathrm{x}$-ray regime at the S K edge and maps of the resonant Auger spectra in the S KLL Auger kinetic energy range have been recorded. Supported with $a b$ initio calculations of the unoccupied electronic structure, these allow us to relate resonances in the absorption cross section to excitations along various directions in the Brillouin zone. We observe anisotropy in the x-ray absorption cross section in polarization directions in plane and out of plane of the crystal. There is also anisotropy in the charge transfer dynamics as inferred from the coherent and noncoherent parts of the resonant Auger spectra. This approach can be generally used to interpret dynamics in unoccupied states, e.g., in layered structure or heterogenous interfaces.
\end{abstract}

DOI: 10.1103/PhysRevB.102.035165

\section{INTRODUCTION}

Transition-metal dichalcogenides (TMDs) are among the candidates for two-dimensional (2D) materials beyond graphene and have shown promise in applications, e.g., for next-generation electronics [1] and in rechargeable batteries [2]. TMDs are a family of layered materials weakly bonded through van der Waals forces with the chemical formula $M X_{2}$ where $M$ is a transition-metal ion and $X$ is a chalcogenide ion. A material belonging to this class is $\mathrm{SnS}_{2}$, a yellow layered crystal, which has been used in applications such as photocatalysis [3], thermoelectrics [4], and optoelectronics [5]. The electronic properties of $\mathrm{SnS}_{2}$, and TMDs in general, change depending on the thickness [6] and geometry [7] of the crystal.

In this study, we use core-hole clock spectroscopy (CHCS) [8] as a probe of charge transfer dynamics and its anisotropic behavior within and out of the plane of singlecrystal $\mathrm{SnS}_{2}$. CHCS uses the finite lifetime of a core-excited state as an internal clock to measure the dynamical processes. Using this technique with excitation energies in the tender $\mathrm{x}$-ray range allows the probe to be both chemically specific and it can probe processes down to percent of the core-hole lifetime (tens of attoseconds) [9].

The core-excited state is reached through the absorption of an x-ray photon, here in the tender energy range (2$10 \mathrm{keV})$. The cross section for $\mathrm{x}$-ray absorption including dependence of the photon's polarization is in the dipole

\footnotetext{
*fredrik.johansson@physics.uu.se
}

Published by the American Physical Society under the terms of the Creative Commons Attribution 4.0 International license. Further distribution of this work must maintain attribution to the author(s) and the published article's title, journal citation, and DOI. Funded by Bibsam. approximation [10]:

$$
\sigma(\omega)=4 \pi^{2} \hbar \omega \alpha \sum_{f}\left|\left\langle f|\hat{\epsilon} \cdot \mathbf{r}| \phi_{S 1 s}\right\rangle\right|^{2} \delta\left(E_{f}-E_{S 1 s}-\hbar \omega\right),
$$

where $\left|\phi_{S 1 s}\right\rangle$ is the atomiclike S $1 s$ core orbital and $|f\rangle$ is the unoccupied states of the system. The dipole operator, $\hat{\epsilon} \cdot \mathbf{r}$, gives rise to selection rules, e.g., the angular momentum quantum number needs to change \pm 1 and that the orbital must lie in the polarization plane. In Figs. 1(a)-1(b) we illustrate the latter with $p$ orbitals lying in or out of plane.

The basis of CHCS is to use such a resonant core excitation [Fig. 1(c)] and monitor the subsequent autoionization processes from states $|f\rangle$. The autoionization can be through a spectator decay, a coherent process where the excited electron stays localized on the excited atom [Fig. 1(d)]. The kinetic energy of the emitted electron is then proportional to the energy of the incoming photon. In the solid state the decay can occur after the excited electron has tunneled away from the atom [Fig. 1(f)]. This is an incoherent process having the same final state as normal Auger emission [Fig. 1(e)]. The kinetic energy of the emitted electron here is independent of the energy of the incoming photon $[9,11]$. The intensity ratio between the coherent, Raman $\left(I_{\text {Raman }}\right)$, and normal Auger $\left(I_{\mathrm{NA}}\right)$ channels is commonly referred to as the Raman-ratio and is used to calculate the charge transfer time $\left(\tau_{\mathrm{CT}}\right)$ using Eq. (2) where $\tau_{1 s}$ is the core-hole lifetime.

$$
\tau_{\mathrm{CT}}=\frac{\sum I_{\mathrm{Raman}}}{\sum I_{\mathrm{NA}}} \tau_{1 s}
$$

In this study we use CHCS to study the components of the sulfur K-edge x-ray absorption spectrum of $\mathrm{SnS}_{2}$. Taking advantage of the sensitivity of CHCS in the tender x-ray regime we map out the unoccupied band structure of the system and relate it to the orbital projection of sulfur. 
(a)

(b)

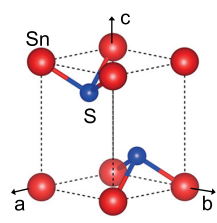

(c)

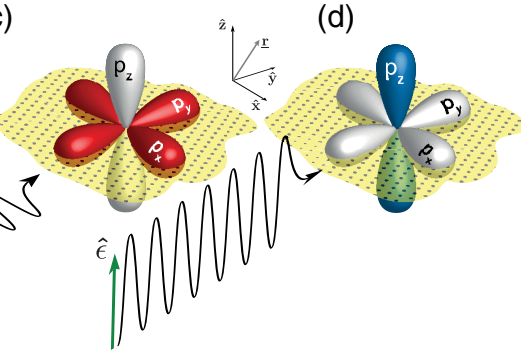

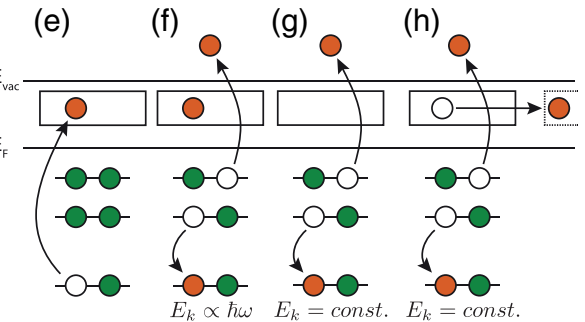

FIG. 1. (a) The unit cell and (b), the first Brillouin zone of $2 \mathrm{H}-\mathrm{SnS}_{2}$. The high symmetry points are indicated by the red dots. (c) and (d) shows the sample geometries and how different polarization directions excite orbitals either in plane or out of plane. (e)-(h) schematically shows the processes of resonant excitation, spectator decay, normal Auger decay and decay after charge transfer and their respective excitation energy dependents.

\section{MATERIALS AND METHODS}

\section{A. Materials}

The $\mathrm{SnS}_{2}$ single crystal (>99.995\%) was purchased from HG Graphene (Groningen, the Netherlands) and was cleaved in situ to ensure a clean surface.

\section{B. Experimental method}

The sample was characterized with hard x-ray photoelectron spectroscopy (HAXPES), resonant Auger spectroscopy (RAS) and X-ray absorption spectroscopy (XAS). The hard $\mathrm{X}$-ray measurements were performed at the KMC- 1 beam line [12] using the high kinetic energy end station, HIKE [13] at Helmholtz-Zentrum Berlin (BESSY II). The beam line is equipped with a double crystal monochromator, optimized for photon energies ranging from $2-12 \mathrm{keV}$. The electron kinetic energy was recorded using a Scienta R4000 hemispherical electron analyzer optimized for high kinetic energies. The XAS was recorded in total florescence yield with a Bruker fluorescence detector. During the measurement the base pressure of the experimental chamber was in the high $10^{-9}$ mbar range. The resonant Auger spectra were measured in resonant Raman conditions, meaning that the photon bandwidth was narrower than the lifetime of the core-excited state [14].

The photon beam was horizontal linearly polarized and perpendicular to the sample. By turning the sample, the polarization can be chosen to be in or out of the plane of the sample. In the case of a 2D-layered crystal, as in $\mathrm{SnS}_{2}$, two of the $p$ orbitals will be in the plane and one out of the plane, a drawing of the geometries can be seen in Figs. 1(c) and 1(d).

\section{Computational method}

First principles calculations based on density functional theory are performed using the projector augmented wavebased (PAW) [15,16] VASP [17,18] code. The generalized gradient approximation in the form of Perdew, Burke, and Ernzerhof (PBE) [19] was used for the exchange-correlation potential. The energy cutoff of the plane waves was set to $520 \mathrm{eV}$. The energy precision of $10^{-5} \mathrm{eV}$ was used for the convergence. The structures were optimized until the maximum Hellmann-Feynman force on each atom becomes smaller than $0.001 \mathrm{eV} / \AA$. The Brillouin zone (BZ) was sampled by a $15 \times 15 \times 15$ Monkhorst-Pack grid. The Grimme's
PBE-D3 method [20] was employed to include the interlayer van der Waals (vdW) interaction. For accurately calculating the electronic band structures, Heyd-Scuseria-Ernzerhof (HSE) hybrid functional within the framework of HSE06 was employed [21].

\section{RESULTS}

\section{A. Sample characterization}

As can be seen in the overview spectra in Figs. 2(a)-2(b) the sample is clean and contains pure $\mathrm{SnS}_{2}$, there are traces of $\mathrm{O} 1 s$ and $\mathrm{C} 1 s$, which are from residual gas from the experimental chamber adsorbed on the sample surface. The $\mathrm{S} 1 s$ spectra in Fig. 2(b) consist of a single sulfur species, and can be described using a single Voigt component and a Shirley background in a least-squares fit. The experimental broadening was determined from fit to $\mathrm{Au} 4 f$ spectra of a gold foil, measured with the same experimental settings, using Voigt line shapes. Using known lifetime broadening, 0.3 and $0.28 \mathrm{eV}$ for the $7 / 2$ and 5/2 components, respectively [22], the experimental broadening was determined to be $0.2928 \mathrm{eV}$. The Lorentzian lifetime broadening $\left(\Gamma_{L}\right)$ of the $\mathrm{S} 1 s$ was determined to be $0.70 \mathrm{eV}$ and using Heisenberg's uncertainty principle, $\tau_{1 s}=\hbar \Gamma_{L}$ where $\hbar$ is the reduced Plank's constant and $\tau_{1 s}$ is the lifetime, the lifetime was calculated to be 0.94 femtoseconds.

The S KLL spectrum in Fig. 2(c) was recorded far above the ionization threshold and shows the normal Auger spectra. The major autoionization channels are visible, the $\mathrm{KL}_{2} \mathrm{~L}_{3}$ $\left({ }^{1} \mathrm{D}_{2}\right), \mathrm{KL}_{2} \mathrm{~L}_{2}\left({ }^{1} \mathrm{~S}_{0}\right), \mathrm{KL}_{1} \mathrm{~L}_{2,3}\left({ }^{3} \mathrm{P}_{2,1,0}\right)$, and $\mathrm{KL}_{1} \mathrm{~L}_{2}\left({ }^{1} \mathrm{P}_{1}\right)[23]$. The bulk plasmon is also visible approximately $19 \mathrm{eV}$ below the ${ }^{1} \mathrm{D}_{2}$ and ${ }^{1} \mathrm{P}_{1}$ [24].

\section{B. X-ray absorption and resonant Auger spectroscopy}

Polarization-dependent x-ray absorption of $\mathrm{SnS}_{2}$ around the $\mathrm{S}$ K-edge resonance is presented in Fig. 2(d) as the fluorescence and electron yields and in Figs. 3(a) and 3(b) the resonant Auger spectrum maps. The fluorescence yield in Fig. 2(d) is a measure of the $\mathrm{x}$-ray cross section for photon absorption detected via the decay channel of the core hole that results in the emission of an X-ray. The x-ray absorption cross section can also be measured via electron yield arising from the radiationless decay of the excited state (lower spectra in 
(a)

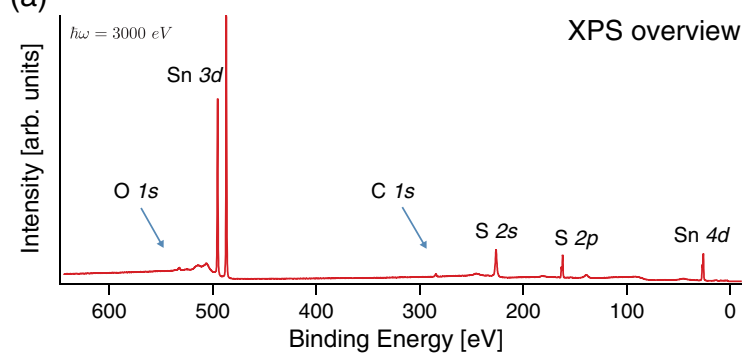

(b)
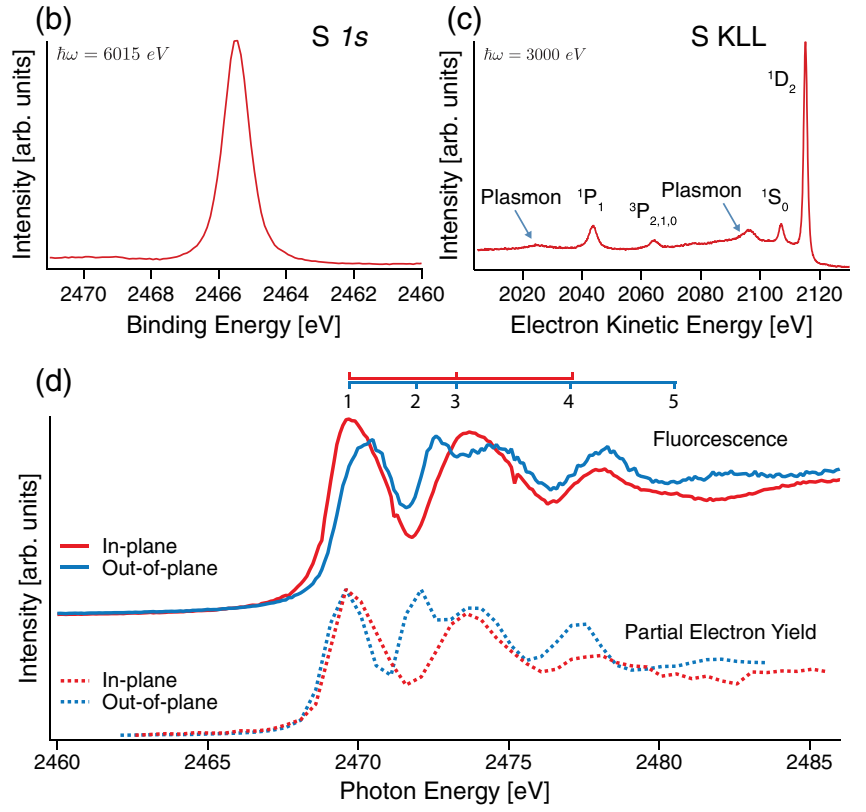

FIG. 2. XPS overview and S $1 s$ spectra as well as the S KLL Auger spectra recorded high above the ionization potential.

Fig. 2(d). The electron yield spectra are the integral horizontal intensity of the maps in Figs. 3(a) and 3(b), respectively. The resonating features seen in the fluorescence yield spectra are also clearly seen in the integral total electron yields for both polarization directions. The main resonances are designated with numbers.

In the resonant Auger maps in Figs. 3(a) and 3(b) each row shows an electron spectrum in the S KLL kinetic energy region. In both maps the $\mathrm{KL}_{2} \mathrm{~L}_{2}\left({ }^{1} \mathrm{~S}_{0}\right)$ and the $\mathrm{KL}_{2} \mathrm{~L}_{3}\left({ }^{1} \mathrm{D}_{2}\right)$ Auger channels are visible at 2107 and $2116 \mathrm{eV}$ kinetic energy respectively. Comparing the in-plane and out-of-plane directions, two things stand out: the extra resonating feature (2) in the out-of-plane map (absent in the in-plane experiment) and that the out-of-plane absorption strength is evenly distributed compared to that observed for the in-plane measurement.

As shown in Fig. 3(c), 2H-SnS 2 is a semiconductor with an indirect band gap of $2.3 \mathrm{eV}$. The valence band maximum (VBM) and the conduction band minimum (CBM) are located close to the $\Gamma$ point and $\mathrm{K}$ point respectively. In order to interpret our experimental results, we have examined the orbital character of the bands relevant to the spectroscopic data. As we are interested in the transition from $1 s$ to $3 p$ bands of sulfur, the contribution of the $p$ orbitals of sulfur atoms projected on to the band structure indicated by the size of the circles is shown in Fig. 3(d). The dominance of different symmetries of sulfur $p$ orbitals at different $k$ point of the Brillouin zone (BZ) is clearly seen in the so-called fat-band plot. For low-energy excitations both out-of-plane $p_{z}$ and in-plane $p_{x}$ and $p_{y}$ orbitals have significant contributions. However, for higher energies, $p_{z}$ orbitals contribute more.

The observed anisotropy in the x-ray absorption cross section can be understood from Eq. (1). The excitation selects directions where the overlap of the final state and the polarization direction is large. Changing the polarization direction, we will highlight different parts of the unoccupied band structure $(|f\rangle)$. The dipole selection rules dictate that angular momentum quantum number must change by unity $(\Delta \ell= \pm 1)$. The unoccupied states that we probe here are thus of $p$ character, since the excitation if from the sulfur $1 s$ orbital. Electronic structure calculations [Fig. 3(d)] also show a different partial density of states with excitation from, e.g., $\Gamma$ to A (out-of-plane) versus $\Gamma$ to $\mathrm{M}$ or $\mathrm{K}$ (in-plane), as in earlier studies [3]. The first excited state represents the bottom of the (a)

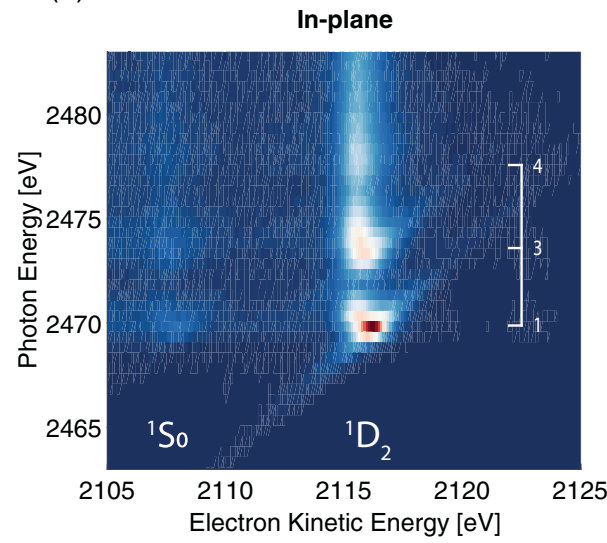

(b)

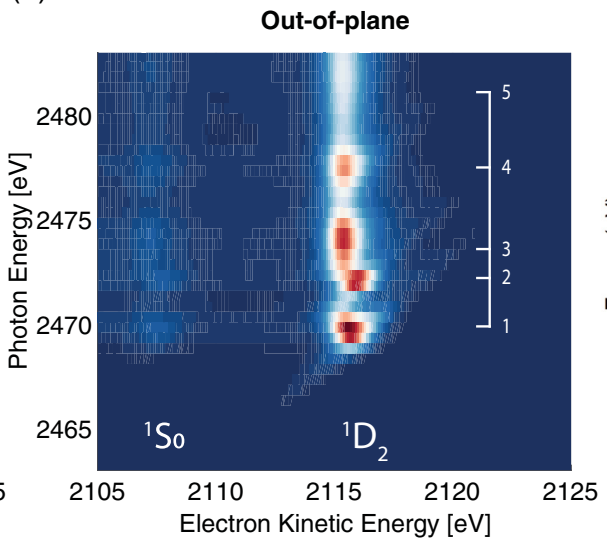

(c)

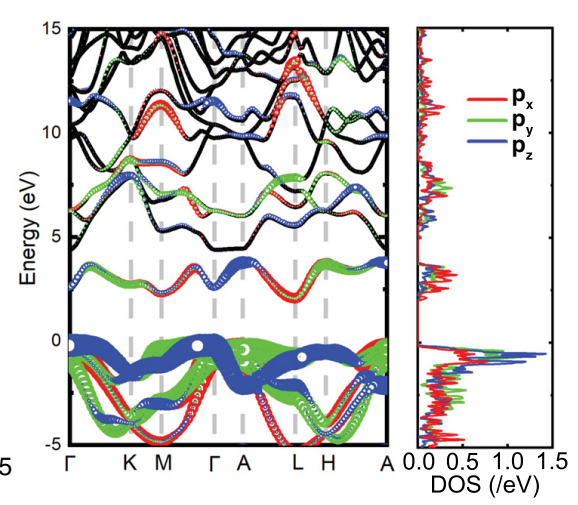

FIG. 3. (a) X-ray absorption and (b) resonant Auger maps in normal and (c) grazing emission, exhibiting differences in the resonance structure. (d) show the atomic orbital projected band structure of $2 \mathrm{H}-\mathrm{SnS}_{2}$, in which the contribution of $p_{x}, p_{y}$, and $p_{z}$ orbitals of $\mathrm{S}$ atoms are illustrated by the size of red, green, and blue circles, respectively. In (e) the corresponding projected densities of states are shown. The zero of energy indicates the valence band maximum (VBM). 
conduction band of the crystal. This can be reached both with in-plane and out-of-plane excitations. However, the intensities are different since $p_{x}, p_{y}$, and $p_{z}$ are represented with different weights in different parts of the band structure [Fig. 3(c)]. The partial density of states for $p_{z}$ is shifted towards higher excitation energies whereas the bottom of the conduction band is dominated by $p_{x}$ at the $L$ point of the BZ.

Intensity at about $2475 \mathrm{eV}$ excitation energy (marked " 2 ") is observed in the out-of-plane series. This intensity is absent in the in-plane map. In the calculated electronic structure it can be seen that $p_{z}$ orbitals along the A-L-H triangle of the Brillouin zone build up a band, which can be excited with polarization perpendicular to the surface. In the spectra this intensity is also dispersing, which means that this is a local decay channel (described in Fig. 1).

Immediately above [2 in Fig. 3(b)] in the out-of-plane map a strong nondispersing streak of significant intensity is situated. In the other map the intensity around [3 in Fig. 3(c)] drops off quickly with increasing excitation energy whereas for out of plane this state is wider. For the in-plane series $p_{x}$ and $p_{y}$ are represented in all directions in the BZ.

The fourth point of the out-of-plane map exhibits both a dispersing Raman channel and a nondispersing normal Augerlike channel. The Raman channel is absent when the excitation is in the plane. For higher excitation energies we can observe a significant intensity difference in the two polarization directions; it is clear that the bands here bunch up since we are close to the ionization potential for the system.

Above we investigated the anisotropy of the x-ray absorption cross section [Eq. (1)] using the electronic structure to identify directions and orbital symmetries. We also identified dispersing (Raman) and nondispersing (Auger-like) parts of the map of resonant Auger spectra. Using the core-hole clock method [Eq. (2)] we can infer how fast the excited electron gets transferred from the site of core excitation. In Fig. 4(a) the partial yields in the different channels are presented. The partial yield is either that of a constant kinetic energy Augerlike charge transfer decay channel or that of a dispersing (constant binding energy) Raman-like local decay channel for the core excited state.

To compare the Auger and Raman yields, the individual Auger spectra have been fitted; first an Auger spectrum above the ionization threshold was fitted to determine the shape and position of the normal Auger peak. It revealed a small asymmetry using a Doniach-Šunić line shape [25], and a Shirleytype background [26]. Each spectrum over the resonance was then fitted with this as the starting point for the Auger part and one, or more, peaks for the Raman part and also with a Shirley background.

The charge transfer times calculated from the yields using Eq. (2) are shown in Fig. 4(b). In the plot, the different resonating features, with the Raman features, are plotted individually. Close to the first resonance the charge transfer is in the femtosecond range and is seen to exhibit the normal exponential decay with higher excitation energy. When increasing the photon energy the charge transfer is faster for the outof-plane geometry indicating that the fastest charge transfer is between the layers in the crystal, this is in contradiction to the previous study by Eads et al. [7], which showed the opposite behavior. The next feature starts at lower energy (a)

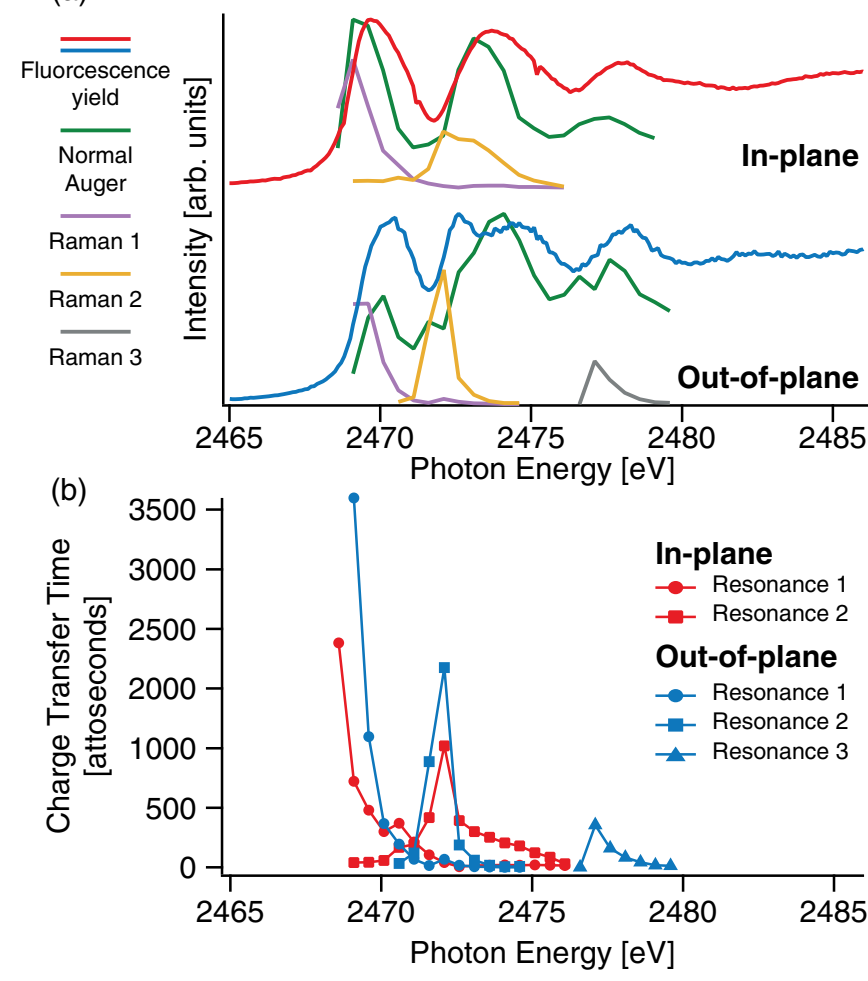

FIG. 4. (a) Comparison of the fluorescence yields absorption cross section and the Auger and Raman yields from the fit of the resonant Auger spectra. (b) Comparison of the charge transfer times for the different resonances.

in the out-of-plane geometry, as indicated in the RAS map (Fig. 3). Comparing the charge transfer at the top of this resonance the in-plane is faster but with increasing excitation energy, the charge transfer in the out-of-plane geometry is much faster.

\section{DISCUSSION}

Looking at the partial yields in the in-plane direction [Fig. 4(a)], the normal Auger contribution alone directly reflects the shape of the XAS spectra. However, in the outof-plane geometry the comparison is not as straightforward; all features in the XAS spectrum can be distinguished but the normal Auger yield alone does not reflect the intensities in the XAS. The shape of the normal Auger yields is very similar to that of a bremsstrahlung-isochromat spectroscopy (BIS) spectra of $\mathrm{SnS}_{2}$ [27]. BIS is a specific case of inverse photoemission spectroscopy where a specific emission energy is monitored and probes unoccupied state by implanting the sample with electrons of a specific energy and recording the emitted photons. This is an indication that resonant Auger spectroscopy and the partial yields are a direct and accurate probe of unoccupied states and can be used as a way to disentangle and explain XAS spectra.

Our result indicates that the charge transfer at the sulfur site in $\mathrm{SnS}_{2}$ on top of the resonances is faster within the layers than between the layers. However, when detuning above the resonance the charge transfer is faster between the layers. 
Earlier work has shown the charge transfer within the layer to be faster, where the study was on charge transfer from the tin sites. Since core-hole clock spectroscopy is chemically specific [9] the charge transfer will be dependent on the chemical surrounding of the atom where the excitation is. In $\mathrm{SnS}_{2}$ the tin atom is in the center of the individual 2D layer and with sulfur atoms both above and below, this means that the neighboring layers are far away. In the case of an excitation on tin the charge transfer should be faster within the layer. In the case of an excitation on sulfur, the closest sulfur atom is in the next layer. The S-S distance in the layer is $3.65 \AA$ and the distance between two layers is $2.94 \AA$ [28].

Looking at the charge transfer time on top of the resonances it gets shorter when the resonances occur at higher excitation energies, from over $3 \mathrm{fs}$ at the first to 350 as at the top of the third. On the resonances the in-plane charge transfer is faster but with increasing excitation energy the dynamics is faster out of plane.

In the ground state of the layered systems considered here each layer is bound via van der Waals forces. However, an electron in the conduction band-here an excited electron in an unoccupied state-may be in an orbital that is shared between layers. Such an orbital couples the electronic states in the different layers to each other. In different directions this coupling is more or less prominent, which is reflected as intensity variations in the unoccupied band structure. Furthermore, depending on the excitation energy we reach different parts of the band structure. This makes it possible to have site, orbital, and directional selectivity in the resonant spectroscopy since we utilize photon energy, $\mathrm{x}$-ray polarization, and sample orientation in concert to highlight certain parts of the unoccupied band structure.

For a heterogeneous interface, $\mathrm{MoS}_{2} / \mathrm{SiO}_{2}$, Garcia-Basabe and coworkers found that the charge transfer times in-plane or out-of-plane upon sulfur $1 s$ excitation differ by a factor of two [29]. For $\mathrm{S} 1 s-3 p$ excitation of chemisorbed sulfur on $\mathrm{Ru}(0001)$, the dynamics is faster in plane rather than out of plane [30]. The propensity for charge transfer thus also depends on whether the neighboring layer has an unoccupied orbital/band, which overlaps with the excited state. It is not sufficient for the layers just to be close to each other. We have also shown this in interface of $\mathrm{MoS}_{2}$ and reduced graphene oxide [31].

\section{CONCLUSIONS}

In conclusion we have shown that charge transfer in $\mathrm{SnS}_{2}$ is anisotropic comparing in-plane and out-of-plane excitation directions through excitations with different x-ray polarization direction. If the polarization is parallel to the surface normal ( $c$ axis of the crystal, out-of-plane direction) we observe three resonances with charge transfer characteristic; in the perpendicular direction (in-plane direction) we observe only two. Using 2D maps of the resonant Auger electron spectra in the S KLL Auger regime and orbital projected electronic structure calculations of the unoccupied states, we can in detail interpret the x-ray absorption cross section and how it depends on polarization direction and orbital character.

The intra- and interlayer coupling is different in different directions for many of the kinds of materials studied here. This makes the study of mono-, bi-, and many-layered variants complex. Typically few-layered systems are those considered for applications in, e.g., optoelectronic systems. In such systems the occupied and unoccupied band structure control the function. Here we have presented core-hole clock spectroscopy that paints a detailed picture of the electronic structure of $\mathrm{SnS}_{2}$.

We suggest that core-hole clock spectroscopy is a general approach to study interfacial charge transfer with not only chemical, but also orbital and directional specificity. For new types of interfaces between 2D materials this kind of spectroscopy provides a window into ultrafast electron dynamics that is made accessible thanks to the high kinetic energy of the outgoing electrons. For devices that have buried monoor few-atomic-layer thin components the specificity of the technique as demonstrated here offers a tool that can study various parts of the interfaces, which are the parts of the devices that provide function.

\section{ACKNOWLEDGMENTS}

A.L. acknowledges the support from the Swedish Research Council (Grants No. 2014-6463 and No. 2018-05336) and Marie Sklodowska Curie Actions (Cofund, Project INCA 600398). O.E. acknowledges support from VR, KAW foundation, ERC, and eSSENCE. B.S. acknowledges Swedish Research Council for financial support and Swedish National Infrastructure for Computing for providing supercomputing allocations. F.J. acknowledges financial support from the $\mathrm{K}$ G Westman Foundation. X.C. would like to thank Chinese Scholarship Council for providing financial support (Grant No. 201606220031). We thank HZB for the allocation of synchrotron radiation beam time and Roberto Felix Duarte for assistance at the KMC-1 beam line.
[1] G. R. Bhimanapati, Z. Lin, V. Meunier, Y. Jung, J. Cha, S. Das, D. Xiao, Y. Son, M. S. Strano, V. R. Cooper et al., ACS Nano 9, 11509 (2015).

[2] T. Momma, N. Shiraishi, A. Yoshizawa, T. Osaka, A. Gedanken, J. Zhu, and L. Sominski, J. Power Sources 97, 198 (2001).

[3] L. A. Burton, T. J. Whittles, D. Hesp, W. M. Linhart, J. M. Skelton, B. Hou, R. F. Webster, G. O’Dowd, C. Reece, D. Cherns et al., J. Mater. Chem. A 4, 1312 (2016).
[4] J. Li, J. Shen, Z. Ma, and K. Wu, Sci. Rep. 7, 1 (2017).

[5] J. Xia, D. Zhu, L. Wang, B. Huang, X. Huang, and X.-M. Meng, Adv. Funct. Mater. 25, 4255 (2015).

[6] J. M. Gonzalez and I. I. Oleynik, Phys. Rev. B 94, 125443 (2016).

[7] C. N. Eads, D. Bandak, M. R. Neupane, D. Nordlund, and O. L. Monti, Nature Commun. 8, 1369 (2017).

[8] P. Brühwiler, O. Karis, and N. Mårtensson, Rev. Mod. Phys. 74, 703 (2002). 
[9] F. O. L. Johansson, M. Ivanovic, S. Svanström, U. B. Cappel, H. Peisert, T. Chassé, and A. Lindblad, J. Phys. Chem. C 122, 12605 (2018).

[10] C. Brouder, J. Phys.: Condens. Matter 2, 701 (1990).

[11] N. Mårtensson, M. Weinelt, O. Karis, M. Magnuson, N. Wassdahl, A. Nilsson, J. Stöhr, and M. Samant, Appl. Phys. A: Mater. Sci. Process. 65, 159 (1997).

[12] F. Schaefers, M. Mertin, and M. Gorgoi, Rev. Sci. Instrum. 78 123102 (2007),

[13] M. Gorgoi, S. Svensson, F. Schäfers, G. Öhrwall, M. Mertin, P. Bressler, O. Karis, H. Siegbahn, A. Sandell, H. Rensmo, W. Doherty, C. Jung, W. Braun, and W. Eberhardt, Nucl. Instrum. Methods Phys. Res., Sect. A: Accel. Spectrometers, Detect. Assoc. Equip. 601, 48 (2009).

[14] M. N. Piancastelli, G. Goldsztejn, T. Marchenko, R. Guillemin, R. K. Kushawaha, L. Journel, S. Carniato, J.-P. Rueff, D. Céolin, and M. Simon, J. Phys. B: At., Mol. Opt. Phys. 47, 124031 (2014).

[15] G. Kresse and D. Joubert, Phys. Rev. B 59, 1758 (1999).

[16] P. E. Blöchl, Phys. Rev. B 50, 17953 (1994).

[17] G. Kresse, J. Non-Cryst. Solids 192-193, 222 (1995).

[18] G. Kresse and J. Furthmüller, Phys. Rev. B 54, 11169 (1996).

[19] J. P. Perdew, K. Burke, and M. Ernzerhof, Phys. Rev. Lett. 77, 3865 (1996).

[20] S. Grimme, J. Comput. Chem. 27, 1787 (2006).
[21] A. V. Krukau, O. A. Vydrov, A. F. Izmaylov, and G. E. Scuseria, J. Chem. Phys. 125, 224106 (2006).

[22] M. Patanen, S. Aksela, S. Urpelainen, T. Kantia, S. Heinäsmäki, and H. Aksela, J. Electron. Spectrosc. Relat. Phenom. 183, 59 (2011).

[23] R. Püttner, D. Céolin, R. Guillemin, R. K. Kushawaha, T. Marchenko, L. Journel, M. N. Piancastelli, and M. Simon, Phys. Rev. A 93, 042501 (2016).

[24] H. Cohen, M. Folman, T. Maniv, R. Brener, E. Lifshitz, and Z. Esterlit, Phys. Rev. B 46, 4446 (1992).

[25] S. Doniach and M. Sunjic, J. Phys. C: Solid State Phys. 3, 285 (1970).

[26] D. A. Shirley, Phys. Rev. B 5, 4709 (1972).

[27] B. Smandek, Y. Gao, T. J. Wagener, J. H. Weaver, F. Lévy, and G. Margaritondo, Phys. Rev. B 37, 4196 (1988).

[28] I. Lefebvre-Devos, J. Olivier-Fourcade, J. C. Jumas, and P. Lavela, Phys. Rev. B 61, 3110 (2000).

[29] Y. Garcia-Basabe, G. G. Parra, M. B. Barioni, C. D. Mendoza, F. C. Vicentin, and D. G. Larrudé, Phys. Chem. Chem. Phys. 21, 23521 (2019).

[30] M. Deppe, A. Föhlisch, F. Hennies, M. Nagasono, M. Beye, D. Sánchez-Portal, P. Echenique, and W. Wurth, J. Chem. Phys. 127, 174708 (2007).

[31] F. O. L. Johansson, U. B. Cappel, M. Fondell, Y. Han, M. Gorgoi, K. Leifer, and A. Lindblad, Phys. Chem. Chem. Phys. 22, 10335 (2020). 